\title{
Completions and Simplicial Complexes
}

\author{
Gilles Bertrand \\ Université Paris-Est, Laboratoire d'Informatique Gaspard-Monge \\ Equipe A3SI, ESIEE Paris
}

\begin{abstract}
In this paper, we first introduce the notion of a completion. Completions are inductive properties which may be expressed in a declarative way and which may be combined. In the sequel of the paper, we show that completions may be used for describing structures or transformations which appear in combinatorial topology. We present two completions, $\left\langle\mathrm{C}_{\mathrm{UP}}\right\rangle$ and $\left\langle\mathrm{C}_{\mathrm{AP}}\right\rangle$, in order to define, in an axiomatic way, a remarkable collection of acyclic complexes. We give few basic properties of this collection. Then, we present a theorem which shows the equivalence between this collection and the collection made of all simply contractible simplicial complexes.
\end{abstract}

Keywords: Completions, simplicial complexes, collapse, simple homotopy, combinatorial topology.

\section{Introduction}

Several approaches have been proposed for the study of topological properties of digital objects in the context of computer imagery:

- The digital topology approach introduced by A. Rosenfeld [5]. Elements of $\mathbf{Z}^{d}$ are linked by some adjacency relations. It is not obvious, in this framework, to define certain topological notions (e.g., a homotopy).

- The connected ordered topological space (COTS) approach introduced by E. Khalimsky [3]. The smallest neighborhood of each point of $\mathbf{Z}^{d}$ differs from one point to another. This allows to recover the structure of a topology.

- The complex cellular approach. An object is seen as a structure consisting of elements of different dimensions called cells. As noticed by V. Kovalevsky [4], it is also possible, with this approach, to recover the structure of a topology.

The underlying topology in the last two approaches corresponds to an Alexandroff space [2]. An Alexandroff space is a topological space in which the intersection of any arbitrary family (not necessarily finite) of open sets is open. There is a deep link between Alexandroff spaces and preorders, i.e., binary relations that are reflexive and transitive. To any Alexandroff space, we may associate a preorder $\leq$ such that $x \leq y$ if and only if $y$ is contained in all open sets that contain $x$. Conversely, a preorder determines an Alexandroff space: a set $O$ is open for this space if and only if $x \in O$ and $x \leq y$ implies $y \in O$.

A map $f$ between two preordered sets $X$ and $Y$ is monotone if $x \leq y$ in $X$ implies $f(x) \leq f(y)$ in $Y$. We have the following result. 


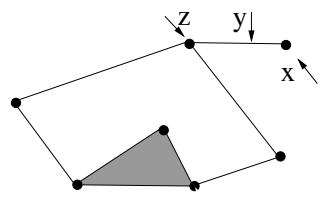

(a)

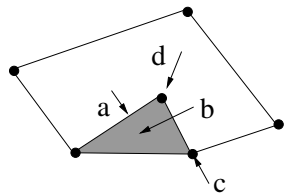

(b)

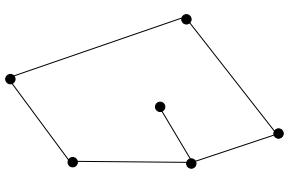

(c)

Fig. 1. (a): A simplicial complex $X$, (b): A complex $Y \subseteq X$, (c): A complex $Z \subseteq Y$.

A map between two preordered sets is monotone if and only if it is a continuous map between the corresponding Alexandroff spaces. Conversely, a map between two Alexandroff spaces is continuous if and only if it is a monotone map between the corresponding preordered sets.

Thus, there is a structural equivalence between Alexandroff spaces and preorders.

Let us consider the (simplicial) objects $X, Y, Z$ depicted Fig. 1. The object $X$ is made of 7 vertices, 8 segments, and 1 triangle. A natural preorder between all these elements is the partial order corresponding to the relation of inclusion between sets. Thus we have $x \leq y$ and $z \leq y$. Let the map $f$ between $X$ and $Y$ be such that $f$ is the identity on all elements of $Y$ and $f(x)=z, f(y)=z$. We note that $f$ is monotone, for example we have $x \leq y$ and $f(x) \leq f(y)$. Thus, $Y$ may be seen as a continuous retraction of $X$ for the corresponding topology. Now, let us try to build a monotone map $g$ between $Y$ and $Z$ such that $g$ is the identity on all elements of $Z$. We see that this is not possible. For example, if we take $g(a)=c, g(b)=c$, we have $d \leq a$, but we have not $g(d) \leq g(a)$. We also observe that it is possible to build such a map between $Y$ and $Y^{\prime}=Y \backslash\{a\}$, but not between $Y^{\prime}$ and $Z$. Thus, in the context of this construction, the classical axioms of topology fail to interpret $Z$ as a continuous retraction of $Y$.

The paper is organized as follows. First we introduce the notion of a completion. Completions are inductive properties which may be expressed in a declarative way and which may be combined. In the sequel of the paper, we show that completions may be used for describing structures or transformations which appear in combinatorial topology. After some basic definitions for simplicial complexes, we give two examples of completions. We recall some definitions relative to the collapse operator which allows to make the two transforms illustrated Fig. 1. We present two completions, $\left\langle\mathrm{C}_{\mathrm{UP}}\right\rangle$ and $\left\langle\mathrm{C}_{\mathrm{AP}}\right\rangle$, in order to define a remarkable collection of acyclic complexes. We give few basic properties of this collection. Then, we present a theorem which shows the equivalence between this collection and the collection made of all simply contractible simplicial complexes.

The paper is self contained. For the sake of place, only few proofs are presented. The other one's will be given in an extended version of the paper. 


\section{Completions}

We introduce the notions of a constructor and a completion. See also Appendix A for more details and for the link with finitary closure operators.

In the sequel, the symbol $\mathbf{S}$ will denote an arbitrary collection. The symbol $\mathcal{K}$ will denote an arbitrary subcollection of $\mathbf{S}$, thus we have $\mathcal{K} \subseteq \mathbf{S}$.

Definition 1. Let $\mathbf{K}$ be a binary relation over $2^{\mathbf{S}}$ and $2^{\mathbf{S}}$, thus $\mathbf{K} \subseteq 2^{\mathbf{S}} \times 2^{\mathbf{S}}$. We say that $\mathbf{K}$ is a constructor (on $\mathbf{S}$ ) if $\mathbf{K}$ is finitary, which means that $\mathbf{F}$ is finite whenever $(\mathbf{F}, \mathbf{G}) \in \mathbf{K}$. If $\mathbf{K}$ is a constructor on $\mathbf{S}$, we denote by $\langle\mathbf{K}\rangle$ the following property which is the completion induced by $\mathbf{K}$ :

$\rightarrow$ If $\mathbf{F} \subseteq \mathcal{K}$, then $\mathbf{G} \subseteq \mathcal{K}$ whenever $(\mathbf{F}, \mathbf{G}) \in \mathbf{K}$.

Let $\mathbf{K}$ be a constructor on $\mathbf{S}$ and let $\mathbf{X} \subseteq \mathbf{S}$. We define:

$$
\mathbf{K}(\mathbf{X})=\cup\{\mathbf{G} \mid \text { there exists }(\mathbf{F}, \mathbf{G}) \in \mathbf{K} \text { with } \mathbf{F} \subseteq \mathbf{X}\} \cup \mathbf{X} \text {. }
$$

We set $\mathbf{K}^{1}(\mathbf{X})=\mathbf{K}(\mathbf{X})$ and $\mathbf{K}^{k}(\mathbf{X})=\mathbf{K}\left(\mathbf{K}^{k-1}(\mathbf{X})\right), k \geq 2$.

We also set $\langle\mathbf{X}, \mathbf{K}\rangle=\cup\left\{\mathbf{K}^{k}(\mathbf{X}) \mid k \geq 1\right\}$.

Let $\langle\mathrm{K}\rangle$ be a property which depends on $\mathcal{K}$. We say that a given collection $\mathbf{X} \subseteq \mathbf{S}$ satisfies $\langle K\rangle$ if the property $\langle K\rangle$ is true for $\mathcal{K}=\mathbf{X}$.

Theorem 1. Let $\mathbf{K}$ be a constructor on $\mathbf{S}$ and let $\mathbf{X} \subseteq \mathbf{S}$. There exists, under the subset ordering, a unique minimal collection which contains $\mathbf{X}$ and which satisfies $\langle\mathbf{K}\rangle$, this collection is precisely $\langle\mathbf{X}, \mathbf{K}\rangle$.

Furthermore, we have $\langle\mathbf{X}, \mathbf{K}\rangle=\cap\{\mathbf{Y} \subseteq \mathbf{S} \mid \mathbf{X} \subseteq \mathbf{Y}$ and $\mathbf{Y}$ satisfies $\langle\mathbf{K}\rangle\}$.

We say that a property $\langle\mathrm{K}\rangle$ is a completion (property) if there exists a constructor $\mathbf{K}$ such that $\langle\mathrm{K}\rangle=\langle\mathbf{K}\rangle$ which means that, for each $\mathcal{K} \subseteq \mathbf{S},\langle K\rangle$ is true if and only if $\langle\mathbf{K}\rangle$ is true. If $\langle\mathrm{K}\rangle$ is a completion and if $\mathbf{X} \subseteq \mathbf{S}$, we write $\langle\mathbf{X}, \mathrm{K}\rangle$ for the unique minimal collection which contains $\mathbf{X}$ and which satisfies $\langle K\rangle$.

Let $\langle\mathrm{K}\rangle$ and $\langle\mathrm{Q}\rangle$ be two completions induced, respectively, by the constructors $\mathbf{K}$ and $\mathbf{Q}$. We see that $\mathbf{K} \cup \mathbf{Q}$ is a constructor and that $\langle K\rangle \wedge\langle Q\rangle$ is the property induced by $\mathbf{K} \cup \mathbf{Q}$, the symbol $\wedge$ standing for the logical "and". Thus, if $\langle K\rangle$ and $\langle\mathrm{Q}\rangle$ are completions, then $\langle\mathrm{K}\rangle \wedge\langle\mathrm{Q}\rangle$ is a completion.

In the sequel of the paper, we write $\langle K, Q\rangle$ for the completion $\langle K\rangle \wedge\langle Q\rangle$. Thus, if $\mathbf{X} \subseteq \mathbf{S}$, the notation $\langle\mathbf{X}, \mathrm{K}, \mathrm{Q}\rangle$ stands for the smallest collection which contains $\mathbf{X}$ and which satisfies $\langle K\rangle \wedge\langle\mathrm{Q}\rangle$.

Remark 1. We may build a counter-example which shows that, if $\langle\mathrm{K}\rangle$ and $\langle\mathrm{Q}\rangle$ are completions, then $\langle\mathrm{K}\rangle \vee\langle\mathrm{Q}\rangle$ is not necessarily a completion, the symbol $\vee$ standing for the logical "or".

A constructor $\mathbf{K}$ is one-to-one if $\operatorname{Card}(\mathbf{F})=\operatorname{Card}(\mathbf{G})=1$ whenever $(\mathbf{F}, \mathbf{G}) \in$ $\mathbf{K}$. If $\mathbf{K}$ is a one-to-one constructor, we set $-\mathbf{K}=\{(\mathbf{G}, \mathbf{F}) \mid(\mathbf{F}, \mathbf{G}) \in \mathbf{K}\}$, the constructor $-\mathbf{K}$ is the inverse of $\mathbf{K}$.

It may be seen that, if $\mathbf{K}$ and $\mathbf{Q}$ are two one-to-one constructors, we have $\langle\mathbf{K}\rangle$ $=\langle\mathbf{Q}\rangle$ if and only if $\langle-\mathbf{K}\rangle=\langle-\mathbf{Q}\rangle$.

A one-to-one completion $\langle\mathrm{K}\rangle$ is a completion induced by some one-to-one constructor $\mathbf{K}$, we write $\langle-K\rangle$ for the constructor induced by $-\mathbf{K}$. By the preceding property, this definition is consistent since it does not depend on the choice of $\mathbf{K}$. 


\section{Basic Definitions for Simplicial Complexes}

A complex or an hypergraph is a finite family composed of finite sets. We denote by $\mathbb{H}$ the collection of all complexes.

Let $X \in \mathbb{H}$. The simplicial closure of $X$ is the complex $X^{-}$such that $X^{-}=$ $\{y \subseteq x \mid x \in X\}$. The complex $X$ is a simplicial complex if $X=X^{-}$. We denote by $\mathbb{S}$ the collection of all simplicial complexes. Observe that $\emptyset \in \mathbb{S}$ and $\{\emptyset\} \in \mathbb{S}$.

Let $X \in \mathbb{S}$. An element of $X$ is a simplex of $X$ or a face of $X$. A facet of $X$ is a simplex of $X$ which is maximal for inclusion.

A simplicial subcomplex of $X \in \mathbb{S}$ is any subset $Y$ of $X$ which is a simplicial complex. If $Y$ is a subcomplex of $X$, we write $Y \preceq X$.

A complex $A \in \mathbb{S}$ is a cell if $A=\emptyset$ or if $A$ has precisely one non-empty facet $x$, we set $A^{\circ}=A \backslash\{x\}$ and $\emptyset^{\circ}=\emptyset$. We write $\mathbb{C}$ for the collection of all cells.

Let $X \in \mathbb{S}$. The dimension of $x \in X$, written $\operatorname{dim}(x)$, is the number of its elements minus one. The dimension of $X$, written $\operatorname{dim}(X)$, is the largest dimension of its simplices, the dimension of $\emptyset$ is defined to be -1 .

If $\mathbb{X} \subseteq \mathbb{S}$, we set:

$$
\mathbb{X}[d]=\{X \in \mathbb{X} \mid \operatorname{dim}(X)=d\} \text { and } \mathbb{X}\langle d\rangle=\{X \in \mathbb{X} \mid \operatorname{dim}(X) \leq d\} .
$$

The ground set of $X \in \mathbb{H}$ is the set $X^{\dagger}=\cup\{x \in X\}$. Let $X, Y \in \mathbb{H}$ such that $X^{\dagger} \cap Y^{\dagger}=\emptyset$. The join of $X$ and $Y$ is the complex $X Y$ such that $X Y=$ $\{x \cup y \mid x \in X, y \in Y\}$. Thus, $X Y=\emptyset$ if $Y=\emptyset$ and $X Y=X$ if $Y=\{\emptyset\}$.

In this paper, if $X, Y \in \mathbb{H}$, we implicitly assume that $X$ and $Y$ have disjoint ground sets whenever we write $X Y$.

If $X \in \mathbb{S}$, we say that $A X$ is a simple cone if $A \in \mathbb{C}[1]$, and a cone if $A \in \mathbb{C}$.

The (reduced) Euler characteristic of $X \in \mathbb{S}$ is the number $\chi(X)$ such that $\chi(X)=\sum\left\{(-1)^{\operatorname{dim}(x)} \mid x \in X\right\}$ if $X \neq \emptyset$, and $\chi(\emptyset)=0$. Note that $\chi(X)$ is equal to the ordinary Euler characteristic minus one.

Let $A \in \mathbb{C}$ and $X \preceq A$. The dual of $X$ for $A$ is the simplicial complex, written $(X ; A)^{*}$, such that $(X ; A)^{*}=\left\{A^{\dagger} \backslash x \mid x \in A \backslash X\right\}$. Thus, we have $(X ; A)^{*}=\left\{x \in A \mid\left(A^{\dagger} \backslash x\right) \notin X\right\}$. For any $A \in \mathbb{C}$, we have the following:

- If $X \preceq A$, then $\left((X ; A)^{*} ; A\right)^{*}=X$.

- If $X \preceq A$ and $Y \preceq A$, then $(X \cup Y ; A)^{*}=(X ; A)^{*} \cap(Y ; A)^{*}$.

- If $X \preceq A$ and $Y \preceq A$, then $(X \cap Y ; A)^{*}=(X ; A)^{*} \cup(Y ; A)^{*}$.

- We have $(\emptyset ; A)^{*}=A$ and $(\{\emptyset\} ; A)^{*}=A^{\circ}$.

In the sequel of this paper, we set $\mathbf{S}=\mathbb{S}$. Thus, we will have $\mathcal{K} \subseteq \mathbf{S}$.

In the next two sections, we give some basic examples of completions on $\mathbb{S}$.

\section{Connectedness}

The family composed of all connected simplicial complexes may be defined by means of completions. We define the one-to-one completion $\langle\mathbf{P A T H}\rangle$ as follows.

$\rightarrow$ If $S \in \mathcal{K}$, then $S \cup C \in \mathcal{K}$ whenever $C \in \mathbb{C}$, and $S \cap C \neq\{\emptyset\}$.

$\langle$ РАTH

We may easily verify that $\langle\mathbf{P A T H}\rangle$ is indeed a (one-to-one) completion. The property $\langle\mathbf{P A T H}\rangle$ is the completion induced by the (one-to-one) constructor:

$$
\text { PAтH }=\{(\{S\},\{S \cup C\}) \mid S \in \mathbb{S}, C \in \mathbb{C} \text { and } S \cap C \neq\{\emptyset\}\} .
$$


We set $\Pi=\langle\emptyset$, РАтн $\rangle$. We say that a complex $X \in \mathbb{S}$ is connected if $X \in \Pi$. Observe that $\mathbb{C} \subseteq \Pi$ since, for any $C \in \mathbb{C}$, we have $C \cap \emptyset=\emptyset \neq\{\emptyset\}$. It may be checked that this definition of a connected complex is equivalent to the classical definition based on paths. Now let us define the completion $\langle\Upsilon\rangle$ as follows.

$\rightarrow$ If $S, T \in \mathcal{K}$, then $S \cup T \in \mathcal{K}$ whenever $S \cap T \neq\{\emptyset\}$.

Again, we may easily verify that $\langle\Upsilon\rangle$ is indeed a completion. We have the following result which shows that $\Upsilon$ provides another way to generate $\Pi$.

Proposition 1. We have $\Pi=\langle\mathbb{C}, \Upsilon\rangle$

A property similar to $\langle\Upsilon\rangle$ has been introduced by J. Serra and G. Matheron who proposed, through the notion of a connection, a new set of axioms for connectedness [15]. The main difference between a connection and $\langle\mathbb{C}, \Upsilon\rangle$ is that a connection may be seen as a "static structure" for modeling connectedness, on the contrary $\Upsilon$ is used in $\langle\mathbb{C}, \Upsilon\rangle$ in a "dynamic way" for generating all elements of $\Pi$. On the other hand, $\Upsilon$ works only for finite objects.

\section{Trees}

A tree is classically defined as a graph which is path-connected and which does not contain any cycle. We give here a definition based on the following one-toone completion $\langle$ TREE $\rangle$.

$\rightarrow$ If $S \in \mathcal{K}$, then $S \cup A \in \mathcal{K}$ whenever $A \in \mathbb{C}\langle 1\rangle$ and $S \cap A \in \mathbb{C}\langle 0\rangle . \quad\langle$ TREe $\rangle$ We set Tree $=\langle\emptyset$, TREE $\rangle$. We say that a complex $X \in \mathbb{S}$ is a tree if $X \in$ Tree.

It may be checked that this definition of a tree is equivalent to the classical one.

Through this example, we observe that completions allow to define a collection in a constructive way rather by the means of properties of this collection.

We can express, in a concise manner, a fundamental property of trees.

Proposition 2. We have Tree $=\langle$ Tree, $-\mathbf{T R E E}\rangle$.

\section{Collapse}

We now present some completions related to the collapse operator introduced by J.H.C. Whitehead [12]. Let us recall a classical definition of collapse.

Let $X \in \mathbb{S}$. We say that a face $x \in X$ is free for $X$ if $x$ is a proper face of exactly one face $y$ of $X$, such a pair $(x, y)$ is said to be a free pair for $X$. If $(x, y)$ is a free pair for $X$, the complex $Y=X \backslash\{x, y\}$ is an elementary collapse of $X$.

We define the one-to-one completion $\langle\mathbf{C O L}\rangle$ :

$\rightarrow$ If $S \in \mathcal{K}$, then $S \cup A B \in \mathcal{K}$ whenever $A \in \mathbb{C}[0], A B \in \mathbb{C}, S \cap A B=A B^{\circ} .\langle\mathbf{C O L}\rangle$

Observe that, if $A B \in \mathbb{C}$, and if $A \in \mathbb{C}[0]$, then necessarily $B \in \mathbb{C}$ or $B=\{\emptyset\}$. If $B=\{\emptyset\}, B^{\circ}$ is defined to be $\emptyset$.

It may be seen that, if $S$ and $S \cup a B$, with $B \neq \emptyset$, fulfill the above conditions, then $S$ is an elementary collapse of $S \cup a B$. Conversely, we may formulate any elementary collapse by such an expression. Thus $\langle\mathbf{C O L}\rangle$ is an alternative definition of collapse. The following is a direct consequence of the previous definitions. 


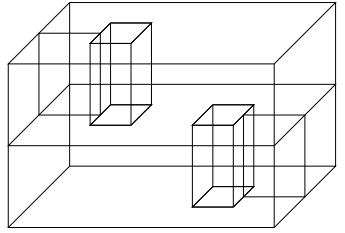

(a)

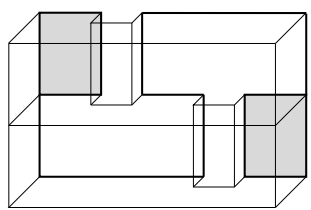

(b)

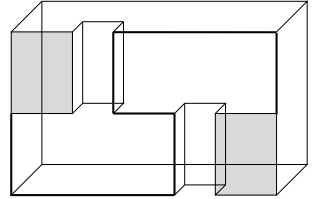

(c)

Fig. 2. (a): A Bing's house $X$ with two rooms, (b): An object $Y \subseteq X$, (c): An object $Z \subseteq X$. We have $X=Y \cup Z$, the object $Y \cap Z$ is outlined in (b) and (c).

Proposition 3. Let $A \in \mathbb{C}, X \preceq A, Y \preceq A$. The complex $X$ is an elementary collapse of $Y$ if and only if $(Y ; A)^{*}$ is an elementary collapse of $(X ; A)^{*}$.

We say that an element of $\langle\emptyset, \mathbf{C O L}\rangle$ is collapsible and that an element of $\langle\emptyset, \mathbf{C O L}$, $-\mathbf{C O L}\rangle$ is simply contractible. If $X, Y \in \mathbb{S}$, we say that $Y$ collapses onto $X$ if $Y \in$ $\langle\{X\}, \mathbf{C O L}\rangle$, and that $Y$ is simple homotopic to $X$ if $Y \in\langle\{X\}, \mathbf{C O L},-\mathbf{C O L}\rangle$.

Let us consider the one-to-one completion:

$\rightarrow$ If $S \in \mathcal{K}$, then $S \cup A \in \mathcal{K}$ whenever $A \in \mathbb{C}$, and $A$ collapses onto $S \cap A$. $\langle$ sim $\rangle$

When $A$ satisfies $\langle\mathbf{s I M}\rangle$, we say that the cell $A$ is simple for $S$. We have $\langle\mathbf{S I M}\rangle=\langle\mathbf{C O L}\rangle$. This completion leads to a notion of simplicity introduced in the context of computer imagery [16] (see also [17], 18]) where an object is often seen as a set of cells (e.g., a set of voxels in 3D) rather than a set of faces.

\section{The Cup/Cap Completions}

We introduce the notion of a dendrite for defining a remarkable collection made of acyclic complexes.

Definition 2. We define the two completions $\left\langle\mathrm{C}_{\mathrm{UP}}\right\rangle$ and $\left\langle\mathrm{C}_{\mathrm{AP}}\right\rangle$ :

$\rightarrow$ If $S, T \in \mathcal{K}$, then $S \cup T \in \mathcal{K}$ whenever $S \cap T \in \mathcal{K}$.

$\rightarrow$ If $S, T \in \mathcal{K}$, then $S \cap T \in \mathcal{K}$ whenever $S \cup T \in \mathcal{K}$.

$\left\langle\mathrm{C}_{\mathrm{UP}}\right\rangle$

We set $\mathbb{R}=\left\langle\mathbb{C}, \mathrm{C}_{\mathrm{UP}}\right\rangle$ and $\mathbb{D}=\left\langle\mathbb{C}, \mathrm{C}_{\mathrm{UP}}, \mathrm{C}_{\mathrm{AP}}\right\rangle$.

Each element of $\mathbb{R}$ is a ramification and each element of $\mathbb{D}$ is a dendrite.

The Bing's house with two rooms [13] is a classical example of an object which is contractible but not collapsible, this object is depicted Fig. 2(a). Let us consider the two complexes $Y$ and $Z$ of Fig. 2(b) and (c). They are such that $X=Y \cup Z$. If $X$ is correctly triangulated, then $Y, Z$, and $Y \cap Z$ are ramifications. Thus, the Bing's house $X$ is a ramification.

It may be easily seen that we have $\langle\emptyset, \mathbf{C O L}\rangle \subseteq\left\langle\mathbb{C}, \mathrm{C}_{\mathrm{UP}}\right\rangle$. Since the Bing's house is not collapsible, this inclusion is strict.

M. Hochster [10] (see also [8] [9]) introduced the notion of a constructible complex. This notion may be expressed using the following completion:

$\rightarrow$ If $S, T \in \mathcal{K}[d]$, then $S \cup T \in \mathcal{K}$ whenever $S \cap T \in \mathcal{K}[d-1], d \geq 0 . \quad\langle$ Cons $\rangle$ A simplicial complex is constructible if it is an element of $\langle\mathbb{C} \cup\{\{\emptyset\}\}$, Cons $\rangle$. 
M. Hachimori [9], 11] showed that the Bing's house with two rooms and the dunce hat 14$]$ are not constructible. Observe that we have $\left\langle\mathbb{C}, \mathrm{C}_{\text {ons }}\right\rangle \subseteq\left\langle\mathbb{C}, \mathrm{C}_{\mathrm{UP}}\right\rangle$. Since the Bing's house is a ramification, the previous inclusion is strict.

With the notion of a buildable complex, J. Jonsson 7] drops the condition for dimension which appears in $\langle$ Cons $\rangle$. The definition of a buildable complex is a recursive definition of what we call a ramification 1 It was shown [7] that any buildable complex is contractible, i.e., is homotopy equivalent to a single point. As far as we know, the above definition for dendrites has never been proposed.

\section{Few Basic Properties}

In this section, we give few basic properties which may be derived directly from the definitions of $\mathbb{R}$ and $\mathbb{D}$ using inductive arguments. Perhaps the simplest property which may be proved in such a way is the following.

Proposition 4. If $X \in \mathbb{D}$, then $\chi(X)=0$.

Proof. We have $\chi(X)=0$ for each $X \in \mathbb{C}$. Since the Euler characteristic is such that $\chi(S \cup T)=\chi(S)+\chi(T)-\chi(S \cap T)$, the result follows by induction.

Let us consider the two completions:

$\rightarrow$ If $S, T \in \mathcal{K}$, then $S \cup T \in \mathcal{K}$.

$\rightarrow$ If $S, T \in \mathcal{K}$, then $S \cap T \in \mathcal{K}$.

Let $\mathbb{X} \subseteq \mathbb{R}$. An element of $\left\langle\mathbb{X}, \mathrm{U}_{\text {NION }}\right\rangle$ is, in general, not necessarily a ramification (nor a dendrite), but this property is true in the following case.

Proposition 5. Let $\mathbb{X} \subseteq \mathbb{R}$ and let $\mathbb{Y}=\left\langle\mathbb{X}, \mathrm{U}_{\mathrm{NION}}\right\rangle$. If $\left\langle\mathbb{Y}, \mathrm{I}_{\mathrm{NTER}}\right\rangle=\mathbb{Y}$, i.e., if $\mathbb{Y}$ satisfies the property $\left\langle\mathrm{I}_{\mathrm{NTER}}\right\rangle$, then we have $\mathbb{Y} \subseteq \mathbb{R}$.

Proof. We set $\mathbb{Y}^{k}=\{X \in \mathbb{Y} \mid \operatorname{Card}(X) \leq k\}$.

i) We have $\mathbb{Y}^{0}=\emptyset$ or $\mathbb{Y}^{0}=\{\emptyset\}$. In both cases $\mathbb{Y}^{0} \subseteq \mathbb{R}$.

ii) Suppose $\mathbb{Y}^{k-1} \subseteq \mathbb{R}$, for some $k \geq 1$. Let $X \in \mathbb{Y}^{k}$. If $X \in \mathbb{X}$, then $X \in \mathbb{R}$. If $X \notin \mathbb{X}$, then there exists $S, T \in \mathbb{Y}$ such that $X=S \cup T$, and $S \nsubseteq T, T \nsubseteq S$. Thus $\operatorname{Card}(S) \leq k-1, \operatorname{Card}(T) \leq k-1$, and $\operatorname{Card}(S \cap T) \leq k-1$. Furthermore, we have $S \cap T \in \mathbb{Y}$. Therefore, by the induction hypothesis, $S, T$ and $S \cap T$ are ramifications, which means that $X$ is a ramification. It follows that $\mathbb{Y}^{k} \subseteq \mathbb{R}$.

Let $A \in \mathbb{C}$ and let $\mathbb{X}=\{B A \mid B \in \mathbb{C}\}$. Since $X A \cup Y A=(X \cup Y) A$, it may be seen that we have $\left\langle\mathbb{X}, \mathrm{U}_{\text {Nion }}\right\rangle=\{X A \mid X \in \mathbb{S}\}$. Furthermore, since $X A \cap Y A=(X \cap Y) A,\left\langle\mathbb{X}, \mathrm{U}_{\text {NION }}\right\rangle$ satisfies the property $\left\langle\mathrm{I}_{\mathrm{NTER}}\right\rangle$. Thus, since $\mathbb{X} \subseteq \mathbb{R}$, the following is a consequence of Prop. 5 ,

Proposition 6. Let $X \in \mathbb{S}$ and $A \in \mathbb{C}$. Then $X A$ is a ramification.

The following fact will be used for the proof of Prop. 8

Proposition 7. Let $A, B \in \mathbb{C}$. Then $(A B)^{\circ}=A B^{\circ} \cup A^{\circ} B$.

${ }^{1}$ We suggest the name "ramification" rather than "buildable complex" since these objects may be seen as natural extensions of trees. 
Proposition 8. Let $X \in \mathbb{S}$ and $A \in \mathbb{C}, A \neq \emptyset$. The complex $X A^{\circ}$ is a dendrite if and only if $X$ is a dendrite.

Proof. If $A \in \mathbb{C}[0]$, we have $A^{\circ}=\{\emptyset\}$ and $X A^{\circ}=X$. Suppose the property is true for any $A \in \mathbb{C}\langle i-1\rangle, i \geq 1$. Let $Y=X A^{\circ}$, with $X \in \mathbb{S}$ and $A \in \mathbb{C}[i]$. Since $i \geq 1$, there exists $B, C \in \mathbb{C}\langle i-1\rangle$, such that $A=B C$. By Prop. 7. we have $X A^{\circ}=X B C^{\circ} \cup X B^{\circ} C$. By Prop. 6, $X B C^{\circ}$ and $X B^{\circ} C$ are dendrites. But $X B C^{\circ} \cap X B^{\circ} C=X B^{\circ} C^{\circ}$. By the induction hypothesis, $X B^{\circ} C^{\circ}$ is a dendrite iff $X B^{\circ}$ is a dendrite, and $X B^{\circ}$ is a dendrite iff $X$ is a dendrite. Thus, by $\left\langle\mathrm{C}_{\mathrm{UP}}\right\rangle$ and $\left\langle\mathrm{C}_{\mathrm{AP}}\right\rangle, Y$ is a dendrite iff $X$ is a dendrite.

We now give a property for duality which will be used in the sequel through two corollaries.

Proposition 9. Let $A, B \in \mathbb{C}$, and let $X, Y \in \mathbb{S}$ such that $X \preceq A, Y \preceq B$. We have $(X Y ; A B)^{*}=A(Y ; B)^{*} \cup B(X ; A)^{*}$.

The following corollary may be obtained from Prop.9. by interchanging $A$ and $B$, and by setting $Y=A$. It shows that the collection of cones is closed by duality. More precisely, if we set $\mathbb{K}=\{A X \mid A \in \mathbb{C}, X \in \mathbb{S}\}$ and $\mathbb{K}^{*}=\left\{(X ; B)^{*} \mid X \in\right.$ $\mathbb{K}, B \in \mathbb{C}, X \preceq B\}$, then we have $\mathbb{K}=\mathbb{K}^{*}$. Note that the collection $\mathbb{C}$ is not closed by duality (By Cor. 2, we have $\left.(A ; A B)^{*}=B^{\circ} A\right)$.

Corollary 1. Let $A, B \in \mathbb{C}$, and let $X \preceq B$. We have $(A X ; A B)^{*}=A(X ; B)^{*}$.

The second corollary may be obtained from Prop. 9 by setting $Y=\{\emptyset\}$.

Corollary 2. Let $A, B \in \mathbb{C}$, and $X \preceq A$. We have $(X ; A B)^{*}=B(X ; A)^{*} \cup B^{\circ} A$.

We see that this last formula allows to calculate the dual of an object in a given space (a cell) from the dual of this object in a smaller space.

Proposition 10. Let $A, B \in \mathbb{C}$, and $X \preceq A$. Then $(X ; A B)^{*}$ is a dendrite if and only if $(X ; A)^{*}$ is a dendrite.

Proof. Let $Y=(X ; A B)^{*}$. By Cor. 2, we have $Y=B(X ; A)^{*} \cup B^{\circ} A$. By Prop. 6. $B(X ; A)^{*}$ and $B^{\circ} A$ are dendrites. Since $B(X ; A)^{*} \cap B^{\circ} A=B^{\circ}(X ; A)^{*}$ and by Prop. 8 , it follows that $Y$ is a dendrite if and only if $(X ; A)^{*}$ a dendrite.

From the very definition of a dendrite, it is clear that, if $X$ is a dendrite, then there exists $A \in \mathbb{C}$ such that $(X ; A)^{*}$ is a dendrite. As a consequence of Prop. 10. we have the following which extends this property for all cells containing $X$.

Proposition 11. If $X \in \mathbb{D}$ and if $X \preceq A$, with $A \in \mathbb{C}$, then $(X ; A)^{*} \in \mathbb{D}$.

\section{Completions and Simple Homotopy}

The following Th. 2 states the equivalence between dendrites and simply contractible complexes. For the sake of space, we give only one part (in fact the easiest part) of the proof. 
Lemma 1. Let $X \in \mathbb{S}$. If $X$ is simply contractible, then $X$ is a dendrite.

Proof. Let $X \in \mathbb{S}$ such that $X$ is simply contractible. Thus, there exists a sequence $\left(X_{0}, \ldots, X_{k}\right)$ such that $X_{0}=\emptyset, X_{k}=X$, and, for each $i \in[1, k]$, either $X_{i}$ is an elementary collapse of $X_{i-1}$ or $X_{i-1}$ is an elementary collapse of $X_{i}$. Let $C \in \mathbb{C}$ such that we have $X_{i} \preceq C$ for each $i \in[0, k]$. The complex $X_{0}$ is a dendrite, suppose $X_{i-1}$ is a dendrite for some $i \geq 1$.

i) If $X_{i-1}$ is an elementary collapse of $X_{i}$, then $X_{i}=X_{i-1} \cup A B$, with $A \in \mathbb{C}[0]$, $A B \in \mathbb{C}, X_{i-1} \cap A B=A B^{\circ}$. By Prop. 6, $A B$ and $A B^{\circ}$ are both dendrites. Thus, by $\left\langle\mathrm{C}_{\mathrm{uP}}\right\rangle, X_{i}$ is a dendrite.

ii) If $X_{i}$ is an elementary collapse of $X_{i-1}$, then, by Prop. 3. $\left(X_{i-1} ; C\right)^{*}$ is an elementary collapse of $\left(X_{i} ; C\right)^{*}$. Furthermore, by Prop. 11. $\left(X_{i-1} ; C\right)^{*}$ is a dendrite. By using the arguments of i) in the dual, we may affirm that $\left(X_{i} ; C\right)^{*}$ is a dendrite. By using again Prop. 11, it follows that $X_{i}$ is a dendrite.

Theorem 2. We have $\mathbb{D}=\langle\emptyset$, COL, - COL $\rangle$. In other words, a simplicial complex is a dendrite if and only if it is simply contractible.

We define the one-to-one completion $\langle\mathbf{D E F}\rangle$ which allows to make homotopic deformations of arbitrary complexes:

$\rightarrow$ If $S \in \mathcal{K}$, then $S \cup D \in \mathcal{K}$ whenever $D \in \mathbb{D}$ and $S \cap D \in \mathbb{D}$.

$\langle$ DEF $\rangle$

Theorem 3. Let $X, Y \in \mathbb{S}$. The complex $Y$ is simple homotopic to $X$ if and only if $Y \in\langle\{X\}, \mathbf{D E F},-\mathbf{D E F}\rangle$.

\section{Conclusion}

We have seen that (one-to-one) completions allow to formulate recursive transformations of objects. More remarkably, completions allow to define structures on objects (e.g., a connection). We introduced two completions, $\left\langle\mathrm{C}_{\mathrm{UP}}\right\rangle$ and $\left\langle\mathrm{C}_{\mathrm{AP}}\right\rangle$, in order to define, in an axiomatic way, a collection of acyclic complexes. We gave a theorem which shows the equivalence between this collection and the collection made of simply contractible simplicial complexes, i.e., complexes which may be transformed to a single point by collapse/anti-collapse operations. Thus, these two axioms may be used for expressing homotopic transforms such as the one illustrated Fig. 1] As we have seen in the introduction, this transformation cannot be interpreted as a continuous one when we consider the preorder associated to the classical axioms of topology.

\section{References}

1. Aczel, P.: An introduction to inductive definitions. In: Barwise, J. (ed.) Handbook of Mathematical Logic, pp. 739-782 (1977)

2. Alexandroff, P.: Diskrete Räume. Mat. Sbornik 2, 501-518 (1937)

3. Khalimsky, E.D.: On topologies of generalized segments. Soviet Math. Doklady 10, 1508-1511 (1969) 
4. Kovalevsky, V.: Finite topology as applied to image analysis. In: Proc. of Comp. Vision Graphics, and Im., vol. 46, pp. 141-161 (1989)

5. Rosenfeld, A.: Digital topology. Amer. Math. Monthly, 621-630 (1979)

6. Tarski, A.: Logic, semantics and metamathematics. Oxford University Press, Oxford (1956)

7. Jonsson, J.: Simplicial Complexes of Graphs. Springer, Heidelberg (2008)

8. Björner, A.: Topological methods. In: Graham, R., Grötschel, M., Lovász, L. (eds.) Handbook of Combinatorics, pp. 1819-1872. North-Holland, Amsterdam (1995)

9. Hachimori, M.: Combinatorics of constructible complexes, PhD Thesis, Tokyo University (2000)

10. Hochster, M.: Rings of invariant of tori, Cohen-Macaulay rings generated by monomials, and polytopes. Ann. Math. 96, 318-337 (1972)

11. Hachimori, M.: Nonconstructible simplicial balls and a way of testing contructibility. Discrete Comp. Geom. 22, 223-230 (1999)

12. Whitehead, J.H.C.: Simplicial spaces, nuclei, and $m$-groups. Proc. London Math. Soc. 45(2), 243-327 (1939)

13. Bing, R.H.: Some aspects of the topology of 3-manifolds related to the Poincaré Conjecture. In: Saasty, T.L. (ed.) Lectures on Modern Mathematics II, pp. 93-128. Wiley, Chichester (1964)

14. Zeeman, E.C.: On the dunce hat. Topology 2, 341-358 (1964)

15. Serra, J.: Image Analysis and Mathematical Morphology, part II: theoretical advances. Academic Press, London (1988)

16. Bertrand, G.: On critical kernels, Comptes Rendus de l'Académie des Sciences. Série Math. I(345), 363-367 (2007)

17. Couprie, M., Bertrand, G.: New characterizations of simple points in 2D, 3D and 4D discrete spaces. IEEE Transactions on PAMI 31(4), 637-648 (2009)

18. Kong, T.Y., Rosenfeld, A.: Digital topology: introduction and survey. In: Proc. of Comp. Vision, Graphics and Image, vol. 48, pp. 357-393 (1989)

19. Welker, V.: Constructions preserving evasiveness and collapsibility. Discrete Math. 207, 243-255 (1999)

20. Kahn, J., Saks, M., Sturtevant, D.: A topological approach to evasiveness. Combinatorica 4, 297-306 (1984)

21. Knaster, B.: Un théorème sur les fonctions d'ensembles. Ann. Soc. Pol. Math. 6, 133-134 (1928)

22. Tarski, A.: A lattice theoretical fixed point theorem and its applications. Pacific J. Math. 5, 285-309 (1955)

23. Kleene, S.C.: Introduction to Meta Mathematics. Van Nostrand, New-York (1962)

24. Lassez, J.-L., Nguyen, V.L., Sonenberg, E.A.: Fixed point theorems and semantics: a folk tale. Information Proc. Letters 14, 3 (1982)

\section{Appendix A: Completions and Closure Operators}

In the sequel, the symbol $\mathbf{S}$ denotes an arbitrary collection.

Let $\gamma$ be a map from $2^{\mathbf{S}}$ to $2^{\mathbf{S}}$, such a map is said to be an operator (on $\mathbf{S}$ ).

If $\gamma$ is an operator on $\mathbf{S}$, we set $\gamma^{1}=\gamma$ and $\gamma^{k}=\gamma \circ \gamma^{k-1}, k \geq 2$. We define

$\widehat{\gamma}$ to be the operator such that, for each $\mathbf{X} \subseteq \mathbf{S}, \widehat{\gamma}(\mathbf{X})=\cup\left\{\gamma^{k}(\mathbf{X}) \mid k \geq 1\right\}$.

Let $\gamma$ be an operator on $\mathbf{S}$. We say that:

- $\gamma$ is extensive if, for all $\mathbf{X} \subseteq \mathbf{S}$, we have $\mathbf{X} \subseteq \gamma(\mathbf{X})$. 
- $\gamma$ is increasing if, whenever $\mathbf{X} \subseteq \mathbf{Y} \subseteq \mathbf{S}$, we have $\gamma(\mathbf{X}) \subseteq \gamma(\mathbf{Y})$.

- $\gamma$ is idempotent if, for all $\mathbf{X} \subseteq \mathbf{S}$, we have $\gamma^{2}(\mathbf{X})=\gamma(\mathbf{X})$.

The operator $\gamma$ is a closure operator (on $\mathbf{S}$ ) if $\gamma$ is extensive, increasing, and idempotent. If $\gamma$ is a closure operator, $\mathbf{X} \subseteq \mathbf{S}$ is closed for $\gamma$ if $\gamma(\mathbf{X})=\mathbf{X}$.

Let $\gamma$ be a closure operator on $\mathbf{S}$. We have the following result, this is a basic property of closure operators:

For any $\mathbf{X} \subseteq \mathbf{S}$, we have $\gamma(\mathbf{X})=\cap\{\mathbf{Y} \subseteq \mathbf{S} \mid \mathbf{X} \subseteq \mathbf{Y}$ and $\gamma(\mathbf{Y})=\mathbf{Y}\}$.

Let $\gamma$ be an operator on $\mathbf{S}$. We say that $\gamma$ is finitary if, for all $\mathbf{X} \subseteq \mathbf{S}$, we have $\gamma(\mathbf{X})=\cup\{\gamma(\mathbf{F}) \mid \mathbf{F} \subseteq \mathbf{X}$ and $\mathbf{F}$ finite $\}$.

Alfred Tarski [6] introduced finitary closure operators (also called "finite consequence operators") as an abstract theory of logical deductions. In this context, the set $\mathbf{S}$ represents a set of statements in some language. Given a subset $\mathbf{X}$ of $\mathbf{S}$, the set $\gamma(\mathbf{X})$ represents the set of all statements that may be deduced from $\mathbf{X}$.

Observe that any finitary operator is increasing. Thus, an operator $\gamma$ is a finitary closure operator if and only if $\gamma$ is extensive, finitary, and idempotent.

We say that an operator $\mathbf{K}$ is a $\Lambda$-operator if $\mathbf{K}$ is extensive and finitary.

The following fixed point theorem is essential for our purpose. See [21 24] for more general fixed point theorems.

Theorem 4. Let $\mathbf{K}$ be a $\Lambda$-operator. Then, for any $\mathbf{X} \subseteq \mathbf{S}, \widehat{\mathbf{K}}(\mathbf{X})$ is a fixed point for $\mathbf{K}$, i.e., we have $\mathbf{K}(\widehat{\mathbf{K}}(\mathbf{X}))=\widehat{\mathbf{K}}(\mathbf{X})$.

Proposition 12. If $\mathbf{K}$ is a $\Lambda$-operator, then $\widehat{\mathbf{K}}$ is a finitary closure operator.

Remark 2. If $\gamma$ is an extensive and increasing operator (not necessarily finitary), then $\widehat{\mathbf{K}}(\mathbf{X})$ is not necessarily a fixed point for $\mathbf{K}$.

Let $\mathbf{K}$ be a binary relation over $2^{\mathbf{S}}$ and $2^{\mathbf{S}}$, thus $\mathbf{K} \subseteq 2^{\mathbf{S}} \times 2^{\mathbf{S}}$. We say that $\mathbf{K}$ is a constructor (on $\mathbf{S}$ ) if $\mathbf{K}$ is finitary, which means that $\mathbf{F}$ is finite whenever $(\mathbf{F}, \mathbf{G}) \in \mathbf{K}$.

Let $\mathbf{K}$ be a constructor on $\mathbf{S}$. We also denote by $\mathbf{K}$ the $\Lambda$-operator such that, for each $\mathbf{X} \subseteq \mathbf{S}$, we have:

$$
\mathbf{K}(\mathbf{X})=\cup\{\mathbf{G} \mid \text { there exists }(\mathbf{F}, \mathbf{G}) \in \mathbf{K} \text { with } \mathbf{F} \subseteq \mathbf{X}\} \cup \mathbf{X} .
$$

We say that the operator $\mathbf{K}$ is the $\Lambda$-operator induced by the constructor $\mathbf{K}$.

Let $\mathbf{K}$ be a $\Lambda$-operator on $\mathbf{S}$, the constructor induced by $\mathbf{K}$ is the constructor $\underline{\mathbf{K}}$ such that $\underline{\mathbf{K}}=\{(\mathbf{F}, \mathbf{K}(\mathbf{F})) \mid \mathbf{F}$ is a finite subset of $\mathbf{S}\}$.

The following result is a direct consequence of the above definitions. It shows that specifying a $\Lambda$-operator is (in a certain sense) equivalent to specifying a constructor.

Proposition 13. Let $\mathbf{\mathrm { K }}$ be a $\Lambda$-operator, and let $\underline{\mathbf{K}}$ be the constructor induced by $\mathbf{K}$. The two $\Lambda$-operators $\underline{\mathbf{K}}$ and $\mathbf{K}$ are equal.

Let $\mathbf{K}$ be a constructor on $\mathbf{S}$, and let $\underline{\mathbf{K}}$ be the constructor induced by the A-operator $\mathbf{K}$. The two $\Lambda$-operators $\mathbf{K}$ and $\underline{\mathbf{K}}$ are equal.

Let $\mathbf{K}$ be a constructor. We say that $\mathbf{K}$ is many-to-one if $\operatorname{Card}(\mathbf{G})=1$ whenever $(\mathbf{F}, \mathbf{G}) \in \mathbf{K}$. 
To each arbitrary constructor $\mathbf{K}$ we may associate a many-to-one constructor $\underline{\mathbf{K}}$, we define $\underline{\mathbf{K}}$ to be $\{(\mathbf{F},\{\mathbf{x}\}) \mid$ there exists $(\mathbf{F}, \mathbf{G}) \in \mathbf{K}$ and $x \in \mathbf{G}\}$. We see that the two $\Lambda$-operators $\mathbf{K}$ and $\underline{\mathbf{K}}$ are equal. Thus, specifying a constructor is equivalent to specifying a many-to-one constructor.

Remark 3. Let $\mathbf{K}$ be a binary relation over $2^{\mathbf{S}}$ and $\mathbf{S}$, thus $\mathbf{K} \subseteq 2^{\mathbf{S}} \times \mathbf{S}$. We say that $\mathbf{K}$ is finitary if $\mathbf{F}$ is finite whenever $(\mathbf{F}, \mathbf{x}) \in \mathbf{K}$. It may be seen that specifying a many-to-one constructor is equivalent to specifying such a relation.

In the sequel, the symbol $\mathcal{K}$ will denote an arbitrary subcollection of $\mathbf{S}$, thus we have $\mathcal{K} \subseteq \mathbf{S}$.

If $\mathbf{K}$ is a constructor on $\mathbf{S}$, we denote by $\langle\mathbf{K}\rangle$ the following property which is the completion induced by $\mathbf{K}$ :

$\rightarrow$ If $\mathbf{F} \subseteq \mathcal{K}$, then $\mathbf{G} \subseteq \mathcal{K}$ whenever $(\mathbf{F}, \mathbf{G}) \in \mathbf{K}$.

Let $\langle\mathrm{K}\rangle$ be a property which depends on $\mathcal{K}$. We say that a given collection $\mathbf{X} \subseteq \mathbf{S}$ satisfies $\langle K\rangle$ if the property $\langle K\rangle$ is true for $\mathcal{K}=\mathbf{X}$.

Thus, if $\mathbf{K}$ is a constructor, a collection $\mathbf{X} \subseteq \mathbf{S}$ satisfies $\langle\mathbf{K}\rangle$ if and only if $\mathbf{K}(\mathbf{X})=\mathbf{X}$.

The following propositions are direct consequences of Th. 4 and Prop. 12. Prop. 14 and 15 are equivalent to Th. 1 given in section 2. The collection $\langle\mathbf{X}, \mathbf{K}\rangle$ of Th. 1 is equal to $\widehat{\mathbf{K}}(\mathbf{X})$.

Proposition 14. Let $\mathbf{K}$ be a constructor on $\mathbf{S}$ and let $\mathbf{X} \subseteq \mathbf{S}$. Then $\widehat{\mathbf{K}}(\mathbf{X})$ is, under the subset ordering, the unique minimal collection which contains $\mathbf{X}$ and which satisfies $\langle\mathbf{K}\rangle$.

Let $\langle\mathrm{K}\rangle$ be a property which depends on $\mathcal{K}$.

If $\mathbf{X} \subseteq \mathbf{S}$, we set $\Delta(\mathbf{X}, \mathrm{K})=\cap\{\mathbf{Y} \subseteq \mathbf{S} \mid \mathbf{X} \subseteq \mathbf{Y}$ and $\mathbf{Y}$ satisfies $\langle\mathrm{K}\rangle\}$.

Proposition 15. If $\mathbf{K}$ is a constructor on $\mathbf{S}$ and if $\mathbf{X} \subseteq \mathbf{S}$, then $\widehat{\mathbf{K}}(\mathbf{X})=$ $\Delta(\mathbf{X}, \mathrm{K})$.

We say that a property $\langle\mathrm{K}\rangle$ is a completion (property) if there exists a constructor $\mathbf{K}$ such that $\langle\mathrm{K}\rangle=\langle\mathbf{K}\rangle$ which means that, for each $\mathcal{K} \subseteq \mathbf{S},\langle K\rangle$ is true if and only if $\langle\mathbf{K}\rangle$ is true.

Proposition 16. Let $\langle\mathrm{K}\rangle$ be a property which depends on $\mathcal{K}$. The property $\langle\mathrm{K}\rangle$ is a completion if and only if, for each $\mathbf{X} \subseteq \mathbf{S}$ :

i) $\Delta(\mathbf{X}, \mathrm{K})$ satisfies $\langle\mathrm{K}\rangle$, and

ii) $\Delta(\mathbf{X}, \mathrm{K})=\cup\{\Delta(\mathbf{F}, \mathrm{K}) \mid \mathbf{F} \subseteq \mathbf{X}$ and $\mathbf{F}$ finite $\}$. 